\title{
Application of rapid dot-ELISA for antibody detection of leptospirosis
}

Leptospirosis, a most common zoonotic disease in the world, presents with varied clinical manifestations like febrile jaundice, pyrexia of unknown origin, aseptic meningitis and hepatorenal failure (Alani et al., 1993). The wide spectrum of symptoms confuses the clinical diagnosis and makes it undependable. The laboratory diagnosis of leptospirosis, a prerequisite for treatment, is usually achieved either by isolation of the causative organisms or by serological evidence indicating recent infection (Werts et al., 2001). Isolation of organisms from clinical samples is confirmatory, but a positive culture result may take up to 6 weeks and therefore it doesn't contribute to early diagnosis. The microscopic agglutination test (MAT) is the reference test for diagnosis and detects antibodies at serovar levels (Cinco et al., 1992).

However, the test is time consuming, requires the use of paired sera (Levett \& Whittington, 1998; Sharma et al., 2000), result interpretation is possible only by experts, can show low sensitivity and involves the use of a battery of at least 12-25 leptospires belonging to different serogroups prevalent in that region (Adler et al., 1980). The maintenance of stock cultures and use of live organisms creates a risk of laboratory-acquired infection (Wautkins \& Zochowski, 1990; Winslow et al., 1997). Several methods have been developed for use in diagnosis of leptospirosis as an alternative to MAT, of which IgM ELISA is the most promising and detects genus-specific antibodies (Levett \& Whittington, 1998).

In the present study, a more convenient version of ELISA, IgM dot-ELISA, was developed for antibody detection, and tested on clinical cases of pyrexia of unknown origin and/or febrile jaundice of systemic leptospirosis and ocular leptospirosis. Standardization of dot-ELISA was performed using six different antigens, namely, whole cell antigen, FTL antigen, sonicated antigen, outer-membrane protein (OMP) antigen, boiled antigen and erythrocyte sensitizing substance (ESS) antigen prepared from Leptospira biflexa Semaranga Patoc 1. The concentration was adjusted to $10^{4}-10^{5}$ organisms $\mathrm{ml}^{-1}$ for whole cell antigen, and for other antigens to $100 \mu \mathrm{g} \mathrm{ml}^{-1}$ in $\mathrm{CO}_{3}-\mathrm{HCO}_{3}$ buffer. These leptospiral antigens were used for coating onto the centre of the nitrocellulose tips of 12 projection combs with the help of a microcapillary tube with approximately 1.5-2.0 $\mu$ l volume. The combs were allowed to dry at room temperature. The unbound sites of the nitrocellulose were blocked with either $5 \%$-defatted dry milk powder solution in PBS or $1 \%$ BSA or $0.05 \%$ casein with incubation at $4{ }^{\circ} \mathrm{C}$ overnight. Combs were rinsed in PBS, and dried and kept in a refrigerator for further use. The hyperimmune sera raised in rabbit against L. biflexa serovar Patoc was diluted starting from $1: 100$ to $1: 3200$ in PBS, and added in the first 6 wells of the row of a 96-well microtitre plate, and similarly, the unimmunized rabbit sera was diluted at the same dilutions and added in the next 6 wells of the same row. Combs were incubated in these wells at room temperature for either $30 \mathrm{~min}$ or $45 \mathrm{~min}$ or $1 \mathrm{~h}$. Combs were washed with PBS containing $0.05 \%$ Tween 20 (PBST) for 5-10 min with five to six changes of wash buffer. Further incubation of the combs was carried out in the wells containing goat anti-rabbit horseradishperoxidase conjugate (Dakopats) at a dilution of 1 : 1000 in PBS for either $30 \mathrm{~min}$ or $45 \mathrm{~min}$ or $1 \mathrm{~h}$ at room temperature. Washing was done with PBST for over 5-7 $\mathrm{min}$ and with five to six changes of buffer. Approximately $3 \mathrm{mg}$ diaminobenzedine and $10 \mu \mathrm{H} \mathrm{H}_{2} \mathrm{O}_{2}$ were added to $5 \mathrm{ml}$ PBS and this was dispensed in the fresh wells of microtitration plate at a volume of $200 \mu \mathrm{l}$ per well. Combs were dipped in this solution for $2-3 \mathrm{~min}$. The reaction was stopped with tap water.

Of the six antigen preparations of $L$. biflexa serovar Patoc tested in dot-ELISA, three antigen preparations, namely whole cell antigen, FTL antigen and ESS produced extensive background reactions with the normal sera (control). Reaction intensity was low with sonicated and boiled antigen preparations, and the results also were not very reproducible. But with OMP clear reaction intensity was observed and the results were reproducible. Background reaction with normal sera was also minimal. To further improve reaction intensity from the background noise, different blocking agents, like $5 \%$ defatted dry milk powder solution, $1 \%$ BSA solution and $0.05 \%$ casein solution, were tested. Out of these, blocking the strips with $0.05 \%$ solution of casein following overnight incubation at $4{ }^{\circ} \mathrm{C}$ produced best results when OMP antigen preparation was utilized. Therefore, for further testing, dot-ELISA was performed using OMP antigen, with casein as the blocking agent, to detect leptospiral antibodies in experimental animals and patient's sera. Of the different time periods tried for antibody and conjugate (anti-human horseradish peroxidase), incubation of strips for $45 \mathrm{~min}$ in each produced good results. Reports also suggest that OMPs are highly specific for reactions to Leptospira serovars, mainly because most of the OMPs are highly conserved among pathogenic Leptospira species, and are expressed in both cultivated organisms and in vivo (Haake et al., 2000). OMPs have been characterized, purified, and even analysed by molecular approaches like PCR and cloning, mainly to study their role in pathogenesis or virulence or as protective immunogens. Their utility as diagnostic antigens has not been evaluated in any of the serological tests. Recently, Haake et al. (2000) cloned and expressed the gene encoding one of the most prominent proteins in the outer envelope of leptospires, LipL32, and have found that the expression of the gene is maximum during mammalian infection, and further 
suggested that this may be useful in the development of a good tool for serological diagnosis of leptospirosis.

In the present study, the result of dot-ELISA with OMP as coating antigen was compared with that of the MAT. Out of the 40 sera samples of systemic leptospirosis from a suspected leptospirosis outbreak, 33 were positive by MAT, among these 30 samples were also found to be positive by IgM dot-ELISA, thus showing a $92.5 \%$ correspondence between the 2 test systems. In the case of 455 sera samples of ocular leptospirosis evaluated, it was found that 117 samples were positive and 283 negative by both MAT and dot-ELISA, 21 samples were positive only by dot-ELISA and 13 samples were positive only by MAT with a 87.9 \% correspondence. Reports from other investigators of a high correlation between the MAT and ELISA (Cumberland et al., 1999; Romero et al., 1998; Sehgal et al., 1999) are in agreement with the observations in the present study.

Antibodies in a significant proportion of patients appear within a few days of onset of symptoms (Silva et al., 1995). It has been reported that the genus-specific antibodies are demonstrable earlier than the serovar-specific microscopic agglutinating antibodies. IgM ELISAs, therefore, are expected to be positive at the early stage of disease (Christie, 1980) and have been found useful for the demonstration of Leptospira antibodies during acute stages of the disease (Terpstra et al., 1985). From the clinical point of view, the ability to detect the infection in the early course of the disease is of extreme importance for initiating appropriate treatment and to avoid serious complications (Sehgal et al., 1999). The simple and convenient form of ELISA, IgM dot-ELISA, reported in this study would be of much value in providing early serological diagnosis as a bedside test not requiring any special equipment or technical expertise. The test is also more economical and rapid, with results obtained within $2 \mathrm{~h}$. A more recently developed LEPTO dipstick and the rapid agglutination tests by the Royal Tropical
Institute, Amsterdam, The Netherlands, also represent easy to use tests enabling bedside diagnosis (Gussenhoven et al., 1997). A multicentric evaluation of these tests may merit their application in routine clinical laboratories.

\section{Acknowledgements}

The authors thank the Director of the Defence Research and Development Establishment for encouragement and support. The Ministry of Defence, Government of India, is acknowledged for the funds provided to undertake this work. R. S. also thanks the Ministry of Defence for her fellowship.

\section{Rashmi Sharma, Urmil Tuteja, Rekha Khushiramani, Jyoti Shukla and Harsh Vardhan Batra}

Division of Microbiology, Defence Research and Development Establishment, Jhansi Road, Gwalior 474 002, India

Correspondence

Rekha Khushiramani

(rekkhushi@yahoo.co.in)

or

Harsh Vardhan Batra

(h_v_batra@rediffmail.com)

Adler, B., Faine, S., Muller, H. K. \& Green, D. E. (1980). Maturation of humoral immune response determines the susceptibility of guinea pigs to leptospirosis. Pathology 12, 529-538.

Alani, F. S. S., Mahoney, M. P., Ormerod, L. P., Wright, P. A. \& Garrues, M. (1993). Leptospirosis presenting as atypical pneumonia, respiratory failure and pyogenic meningitis. J Infect 27, 281-283.

Christie, A. B. (1980). Leptospiral infections. In Infectious Disease, Epidemiology and Clinical Practice, 3rd edn., pp. 848-867. London: Churchill Livingstone.

Cinco, M., Balanzin, D. \& Banji, E. (1992). Evaluation of an immunoenzymatic test (ELISA) for the diagnosis of leptospirosis in Italy. Eur J Epidemiol 8, 677-682.

Cumberland, P. C., Everard, C. O. R. \& Levett, P. N. (1999). Assessment of the efficacy of the IgM enzyme linked immunosorbent assay (ELISA) and microscopic agglutination test (MAT) in the diagnosis of acute leptospirosis. Am J Trop Med Hyg 61, 731-734.
Gussenhoven, G. C., van der Hoorn, M. A., Goris, M. G., Terpstra, W. J., Hartskeerl, R. A., Mol, B. W., van Ingen, C. W. \& Smits, H. L. (1997). LEPTO dipstick, a dipstick assay for detection of Leptospira-specific immunoglobulin $\mathrm{M}$ antibodies in human sera. J Clin Microbiol 35, 92-97.

Haake, D. A., Chao, G., Zuerner, R. L., Barnett, J. K., Barnett, D., Mazel, M. K., Matsunaga, J., Levett, P. N. \& Bolin, C. A. (2000). The leptospiral outer membrane protein LipL32 is a lipoprotein expressed during mammalian infection. Infect Immun 68, 2276-2285.

Levett, P. N. \& Whittington, C. U. (1998). Evaluation of the indirect haemagglutination assay for diagnosis of acute leptospirosis. J Clin Microbiol 36, 11-14.

Romero, E. C., Caly, C. R. \& Yasuda, P. H. (1998). The persistence of leptospiral agglutinins titres in human sera diagnosed by the microscopic agglutination test. Rev Inst Med Trop Sao Paulo 40, 183-184.

Sehgal, S. C., Vijyachari, P., Sharma, S. \& Sugunan, A. P. (1999). LEPTO dipstick: a rapid and simple method for serodiagnosis of acute leptospirosis. Trans R Soc Trop Med Hyg 93, 161-164.

Sharma, R., Tuteja, U. \& Batra, H. V. (2000). Use of locally isolated saprophytic Leptospira strain for serological testing of human leptospirosis. $J$ Commun Dis 32, 185-189.

Silva, M. V., Camargo, E. D., Batista, L., Vaz, A. J., Brandao, A. P., Nakamura, P. M. \& Nergrao, J. M. (1995). Behaviour of specific IgM, IgG and IgA class antibodies in human leptospirosis during the acute phase of the disease and during convalescence. J Trop Med Hyg 98, 268-272.

Terpstra, W. J., Ligthart, G. S. \& Schoone, G. J. (1985). ELISA for the detection of specific IgM and IgG in human leptospirosis. J Gen Microbiol 131, 377-385.

Wautkins, S. A. \& Zochowski, W. J. (1990). Leptospira. In ELISA in the Clinical Microbiology Laboratory, pp. 225-237. Edited by T. G. Wreghitt \& P. Morgan-Kapner. London: Public Health Laboratory Service.

Werts, C., Tapping, R. I., Mathison, J. C., Chuang, T.-H., Kravchenko, V., Saint Girons, I., Haake, D. A., Godowski, P. J., Hayashi, F. \& other authors (2001). Leptospiral lipopolysaccharide activates cells through a TLR2-dependent mechanism. Nat Immunol 2, 346-352.

Winslow, W. E., Merry, D. J., Pirc, M. L. \& Devine, P. L. (1997). Evaluation of a commercial enzyme-linked immunosorbent assay for the detection of immunoglobulin $\mathrm{M}$ antibody in diagnosis of human leptospiral infection. J Clin Microbiol 35, 1938-1942. 